\title{
Determining micro droplet profile using internal reflection interference
}

\author{
Iltai Isaac Kim*, Yang Lie, and Jaesung Park \\ Texas A\&M University-Corpus Christi, 6300 Ocean Dr. Corpus Christi, TX 78412-5797, USA
}

\begin{abstract}
We introduce an optical diagnostics to determine the dual-surface profile of liquid droplet using internal reflection interferometry. A coherent laser beam is internally reflected on the air/liquid interface of a sessile droplet placed on a prism-based substrate to produce an interference fringe on a screen far from the substrate. The reflected laser rays consist of the reflection from the center spherical droplet profile and the one from the lower hyperbola-like droplet profile. The reflected rays are interfered each other to form the interference fringes. Ray tracing simulation is conducted using a custom-designed computer program. The simulation shows that the interfering rays reflected near the inflection point produce the outer-most fringes of the concentric interference pattern on the screen, and the reflected rays from the apex of the spherical profile and the contact line of the lower hyperbola-like profile construct the fringes at the center of the interference patterns. The simulated results are compared with the experimental observation to show a good agreement in the number and the location of the fringes and the radius of the outer-most-fringe where the number of the fringes is dependent on the droplet thickness and the radius of the fringe depends on the contact angle of the droplet. This result provides a new measurement technique to determine the morphological features of very small microdroplet such as the thickness ( $<$ a few micron thickness), the contact angle ( $<$ a few degree), and the dualsurface profile.
\end{abstract}

KEY WORDS: Internal reflection interference, Micro droplet, Dual-surface profile, Droplet height, Contact angle, Precursor film

\section{INTRODUCTION}

When microcontact printing $(\mu \mathrm{CP})$ is applied by a stamp with square or circular protrusions, an array of minute droplets can be printed on a target substrate $[1,2]$. The surface profile of printed droplet is characterized with nonmonotonic geometrical aspects such as spherical cap, precursor film, and inflection line [3,4]. The geometrical profile of individual droplet is primarily influenced by the surface energy difference between liquid and substrate. After releasing the stamp from the substrate, the contact line of droplet is expected to recede until the droplet forms a certain shape at an equilibrium state. The resultant droplet forms a dual-profile with an inflection line that partitions a droplet surface geometrically [5]. The upper part of the droplet surface covered from its apex to inflection line takes a nearly spherical-cap shape like a convex lens. From the droplet's edge to inflection line, conversely, the droplet surface shapes in concave upward, which is formed with a foot region and a precursor film. It is an important procedure to verify the geometric shape of droplet surface in some applications such as micro lens array [6], the evaporation or condensation of sessile droplets [7], and the spreading of droplet [8]. There have been various methods of droplet profile measurement based on goniometry [9], interferometry [10-12], total internal reflection fluorescence microscopy (TIRFM) [13], atomic force microscopy (AFM) [14], frustrated total internal reflection (FTIR) [15], and internal reflection [11]. Especially, microscopy technique was used to measure droplet profiles with very thin height and low contact angle; Fizeau interferometry [12] and internal reflection [11].

*Corresponding Author: ikim@tamucc.edu 
In this paper, we introduce a simple optical method to determine the thin height s and low contact angle of micro droplets using internal reflection configuration without complicated microscopy to produce interference fringes depending on the droplet profiles. Existing studies based on reflection and transmission interference [10, 16] show thick droplet height and high contact angles. The proposed method in this study based on internal reflection experimentally demonstrates it effectively measures the droplet height from 1.7 micron to 16 micron with the contact angle from 2.5 to 17 degree. The outcome of this research shows that it can effectively detect the height of thin droplet as small as $\sim 1$ micron and the contact angle as low as $\sim 1$ degree.

To characterize interference fringe patterns, a ray-tracing simulation program is developed based on Excel software with visual basic macro that could provide details of the droplet profiles. A variety of droplets are printed on different substrates with different oleophobicities. A PDMS stamp is used to make micro droplets. The proposed technique based on internal reflection interference can be used effectively in determining the morphological features of micro droplet such as the thickness, contact angle, the precursor film profile, and others.

\section{THEORY}

\subsection{Dual profile of droplet surface}

A sessile droplet is formed with two parts in its surface profile. One is an upper profile that is a macroscopic dorm placing on the apex of droplet $[3,16]$. The other is a lower profile that is mesoscopic film attached at the contact line of droplet. Both profiles are connected along an inflection line (Fig. 1). The dual droplet profile is defined with several parameters such as droplet's height (h), droplet's radius (r), the radial (a) and the axial (b) coordinates of inflection line, and the thickness of precursor film. In this study, we presume that the upper profile shape as a spherical cap and the lower one would be a hyperbola curve. The contact angle of droplet is defined as the slope of droplet surface at the inflection point. The profile of spherical cap can be obtained with droplet height, the coordinates of inflection point, and a contact angle that is associated with the curvature of spherical cap. The profile of hyperbola curve $(\mathrm{Va}$ and $\mathrm{Vb})$ is obtained from the thickness $(\mathrm{t} \sim 100 \mathrm{~nm})$ of precursor film and the coordinates $(a$ and $b$ ) of inflection point. The precursor film ranges from a few tens $\AA$ up to several hundred nanometers according to thermodynamic equilibrium condition of droplet on a substrate [4].

\subsection{Internal reflection and interference fringes}

The interference fringe is made by the internal reflection (IR) system with an equilateral triangle prism (SF10, $n$ $=1.732$ at $633 \mathrm{~nm}$ ). The optical ray tracing about total internal reflection on a droplet surface is illustrated in Figure $1 \mathrm{~b}$. A droplet is placed on the upper side of prism. A He-Ne laser $(\lambda=633 \mathrm{~nm})$ is used as a light source to produce the interferometric fringes of light on a screen. A coherent, parallel beam $(w=0.8 \mathrm{~mm})$ of a laser is incident on the left side of prism and reflects on the upper surface of liquid droplet. Total internal reflection associated with a liquid droplet involves the optical aberration of reflection beam due to the curved profile of droplet surface. The laser beam reflected on the droplet surface is not parallel any more while it passes out through the right side of prism. Eventually, due to the dual profile of droplet surface, the laser beam arrives at different coordinates on a screen according to the position of droplet surface on which it reflects. The interference phenomenon occurs when two optical rays reflected on the upper and lower profiles meet at the same location of screen. The intensity of interfered light depends on the phase difference between both rays, which changes periodically from the center to the out-most of light illumination. The consequential interfered light appears as the concentric fringe pattern.

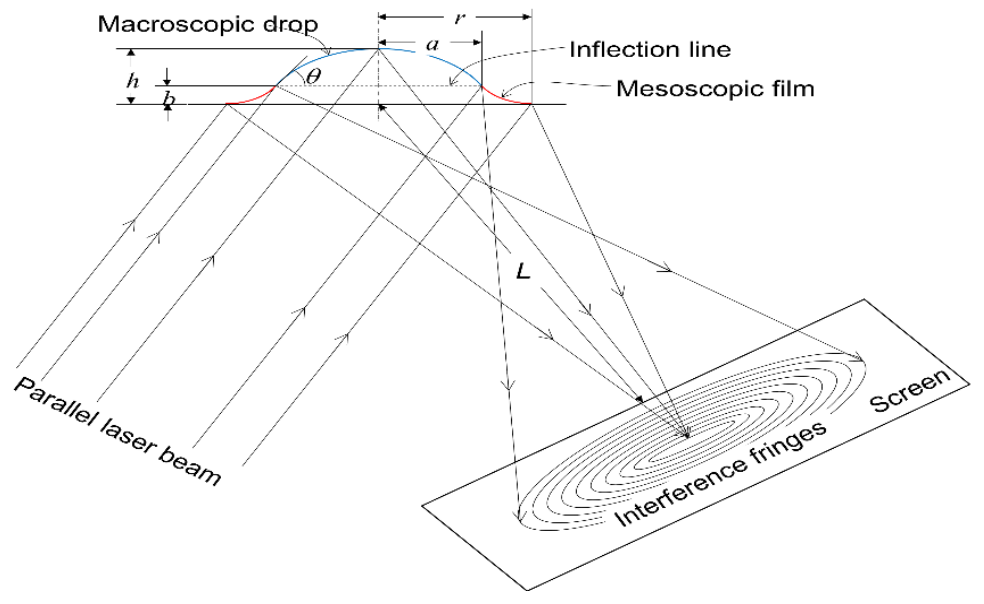


Fig. 1 The dual droplet profile of micro droplet that produces the interference fringes on screen

\section{RESULTS}

\subsection{Microcontact printing with a PDMS stamp}

Microcontact printing $(\mu \mathrm{CP})[1,2,17]$ was applied to form an array of sessile droplets on a glass surface. This method was performed by a polydimethylsiloxane (PDMS) stamp which transferred sample fluid onto a pristine substrate by contact manner. Soft lithography was applied to fabricate the PDMS stamp having an array of micro pillars, which was conventionally operated with a pattern mask, a micro UV aligner, a hot plate, and some chemicals such as Sylgard 184 silicone elastomer (Dow Corning Inc.) and SU-8 photoresist (Microchem Corp.) $[17,18]$. We made a stamp having a square protrusion with the widths of $200 \mu \mathrm{m}$ and the height of $200 \mu \mathrm{m}$.

Fig. 2 presents the representative side-views of droplets transferred by two methods on different coating surfaces. We tried to generate tiny droplets with low contact angle since small, thin droplets make distinct fringe patterns on screen. The volume of droplet is proportional to the amount of sample fluid being pendant on the tip of stamp protrusion or syringe needle. In addition, it can alter along the different adhesion forces of sample fluid on substrate. The diameter (radius) of the droplet is somewhat accurate in the measured data, but the thickness (height) and the contact angle seem to be quite different.
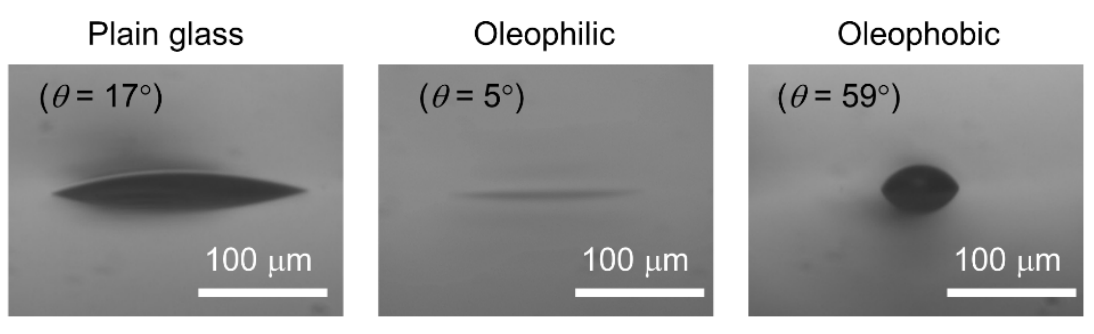

Fig. 2 Different microdroplet profiles on the different coating conditions

\subsection{Internal interference fringes and analysis}

Fig. 3 shows the photographic images for the microdroplets in Fig. 2 on the plain and oleophilic glasses, respectively. Note that the microdroplet on the oleophobic coated glass doesn't show the formation of the interference fringes because of its thick profile which is also verified during the simulation, indicating there is no dual profiles. The images are captured at the incidence angle of $43.2^{\circ}$. The simulation generates the same number of fringes with the corresponding locations according to the ray-tracing scheme.

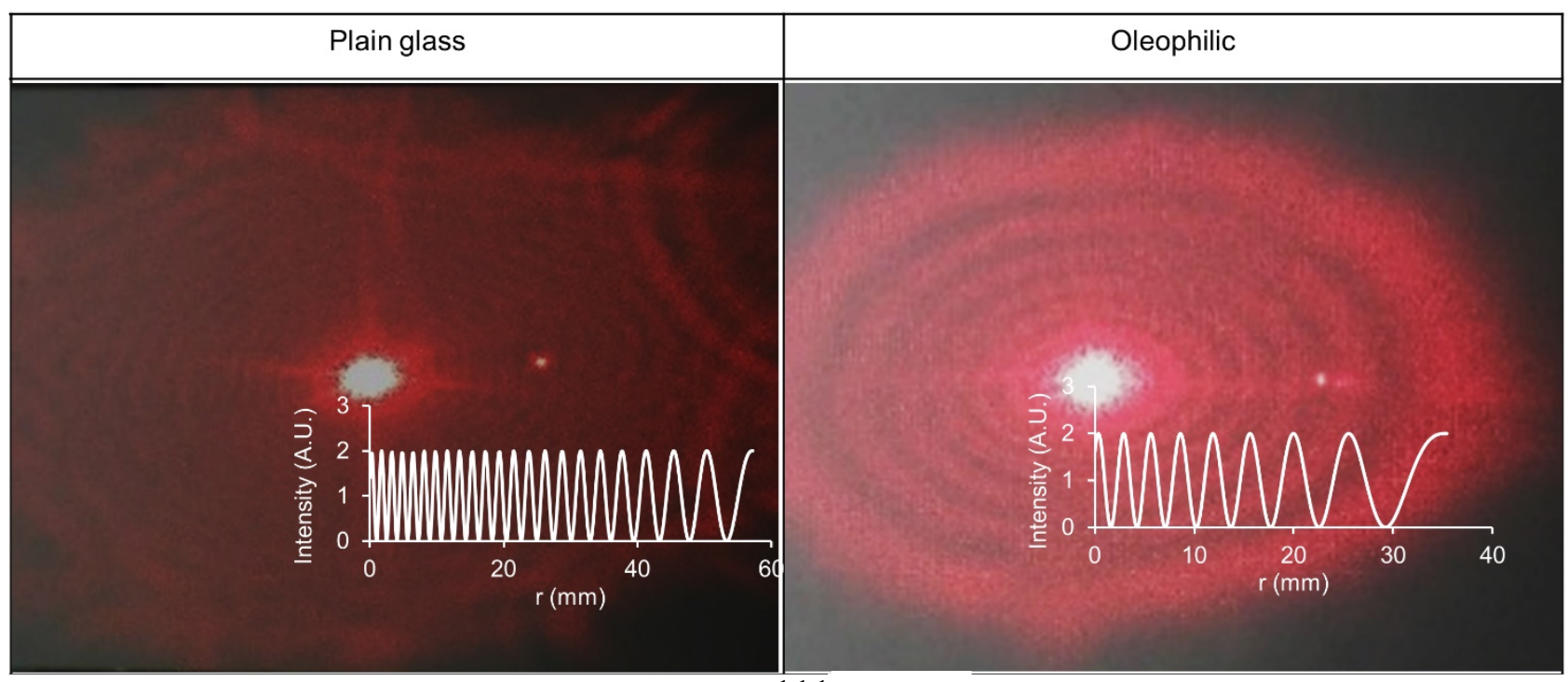


Fig. 3 The photographic images for the interference fringes on the plain and oleophilic coating glasses. The figure also shows the simulated interference fringes with a good agreement.

\section{CONCLUSIONS}

In this presentation, we introduced a new diagnostic technique to measure the morphological features of micro droplets placed on a glass substrate. This method is based on the principle of internal reflection interferometry. A coherent laser beam reflected on the micro droplet produces interference fringes on a far-field screen. This interference fringes care caused by the dual profile characteristics of the microdroplets. The ray tracing was employed to simulate the produced fringes characteristics and showed a very good agreement between the experiment and simulation. The outcome of this study shows the morphological features of micro droplets such as the thickness and the contact angle can be effectively determined by observing the interference fringe formation. In additions, this research can contribute to determine the edge profile of the micro droplets covering the precursor film, which is known to be too small less than a micron to be monitored by the existing visualization technique. The following research is currently under way by combining with the recently developed aperture total internal reflection (A-TIR) technique [17].

\section{ACKNOWLEDGMENT}

This study was supported by a research grant from CEKO Co., LTD and a Texas Comprehensive Research Fund (TCRF) grant from the Texas A\&M University-Corpus Christi Division of Research, Commercialization and Outreach.

\section{REFERENCES}

[1] Alom Ruiz, S. and C.S. Chen, Microcontact printing: A tool to pattern. Soft Matter, 3(2), p. 168-177 (2007).

[2] András, P., R.D. N., and H. Jurriaan, Microcontact Printing: Limitations and Achievements. Advanced Materials, 21(22), p. 2257-2268 (2009).

[3] Mechkov, S., A.M. Cazabat, and G. Oshanin, Post-Tanner stages of droplet spreading: the energy balance approach revisited. Journal of Physics: Condensed Matter, 21(46), p. 464131 (2009).

[4] Popescu, M.N., et al., Precursor films in wetting phenomena. Journal of Physics: Condensed Matter, 24(24): p. 243102 (2012).

[5] Fabiano, G.W., O.E.d.S. Luis, and C.P. Paulo, Modeling and simulation of the fluid-solid interaction in wetting. Journal of Statistical Mechanics: Theory and Experiment, 6, p. P06008 (2009).

[6] Zhu, X., et al., Fabrication of multi-scale micro-lens arrays on hydrophobic surfaces using a drop-on-demand droplet generator. Optics \& Laser Technology, 66, p. 156-165 (2015).

[7] Poulard, C., O. Bénichou, and A.M. Cazabat, Freely Receding Evaporating Droplets. Langmuir, 19(21), p. 8828-8834 (2003).

[8] Bonn, D., et al., Wetting and spreading. Reviews of Modern Physics, 81(2), p. 739-805 (2009).

[9] Lamour, G., et al., Contact Angle Measurements Using a Simplified Experimental Setup. Journal of Chemical Education, 87(12), p. 1403-1407 (2010).

[10] Quo, K.H., T. Uemura, and W.-J. Yang, Reflection-interference method to determine droplet profiles. Applied Optics, 24(16), p. 26552659 (1985).

[11] Campbell, J. M. and Christenson, H. K., Dynamic measurement of low contact angles by optical microscopy. Applied Materials and Interfaces, 10(19), p. 16893-16900 (2018).

[12] Stocklhuber, K.W, Radoev B., and Schulze, H.J., Some new observations on line tension of microscopic droplets. Colloids and Surfaces, 156, p. 323-333 (1999).

[13] Franken, M.J.Z., C. Poelma, and J. Westerweel, Nanoscale contact line visualization based on total internal reflection fluorescence microscopy. Optics Express, 21(22), p. 26093-26102 (2013). 
[14] Aston, D.E. and J.C. Berg, Quantitative Analysis of Fluid Interface-Atomic Force Microscopy. Journal of Colloid and Interface Science, 235(1), p. 162-169 (2001).

[15] Shirota, M., et al., Measuring thin films using quantitative frustrated total internal reflection (FTIR). The European Physical Journal E, 40(5), p. 54 (2017).

[16] Zhang, N. and D.F. Chao, Caustics and caustic-diffraction in laser shadowgraphy of a sessile drop and identification of profile near contact line. Optics \& Laser Technology, 35(3), p. 155-161 (2003).

[17] J. S. Park, Y. Lie, H. J. Kim, H. C. Kim, and I. Kim, Development of aperture total internal reflection (A-TIR) for micro droplet and fingerprinting patterns characterization, Optics Communications, 453, 124414 (2019)

[18] Xia, Y. and G.M. Whitesides, Soft Lithography. Angewandte Chemie International Edition, 37(5), p. $550-575$ (1998). 